\title{
High quality model for compression of medical images in telemedicine
}

\author{
M. Moorthi ${ }^{1}$ and R. Amutha ${ }^{2}$ \\ ${ }^{1}$ Department of Electronics and Communication Engineering, Sri Chandrasekharendra Saraswathi Viswa Mahavidyalaya \\ University, Kanchipuram, India. \\ ${ }^{2}$ Department of Electronics and Communication Engineering, SSN College of Engineering, Chennai, India.
}

Accepted 26 September, 2013

\begin{abstract}
Generally lossless compression should be used for ROI (Region of Interest) and lossy compression should be used for ROB (Region of Background) with a lower quality. In existing system, Region of Interest (ROI) is selected manually, but ROI is selected automatically in the proposed method, preprocessing is done to improve the visual quality of the image. Segmentation is carried out accurately and efficiently using region growing followed by morphological processing method. The features are extracted and classification is done in medical image using Fuzzy logic. ROB part of an image is compressed using SPIHT (Set Partition In Hierarchical Tree) algorithm in near lossless manner. Finally the ROI is superimposed in compressed Non ROI (ROB) image. This method improves the compression ratio and increase the PSNR value compared to existing method. The proposed method is used for implementations of teleradiology and digital picture archiving and communications (PACS) systems practically.
\end{abstract}

Key words: Image compression, segmentation, wavelet decomposition, fuzzy logic , decompression, compression ratio.

\section{INTRODUCTION}

A new medical image compression technique is required for the reduction in diagnostic analysis cost, storage cost and transmission time without affecting quality of the image. Medical science grows very fast and hence each hospital needs to store high volume of data about the patients. And medical images are one of the most important data about patients. The main purpose of telemedicine is to enhance health care delivery to a wider population. This telemedicine technology supports the transfer of pathological and imaging reports of patients across the telemedicine networks, so as to provide consultation by specialists located in geographically different locations. The integration of mobile communication and biomedical instrumentation technology plays an important role in telemedicine as doctors away from the system can also get the health status of their critical patients. To improve the quality of decision making, image segmentation algorithms can be developed which take medical images as input and segment them to produce a well defined area in that image. The medical images have to be transmitted across telemedicine network to remote medical centre for diagnosis. In this connection, effective lossless compression algorithms can be developed which result in saving of storage space and better utilization of bandwidth and speed of data transmission. The regions of interest (ROI) based approach (Joan et al., 2011; Liu and Fan, 2003) is used to compress medical images at particular compression rate. The vascular image consists of the primary region of interest (PROI), the secondary region of interest (SROI) and the background. The region growing algorithm is implemented to detect SROI automatically. The seed point for region growing method is PROI of an image. 


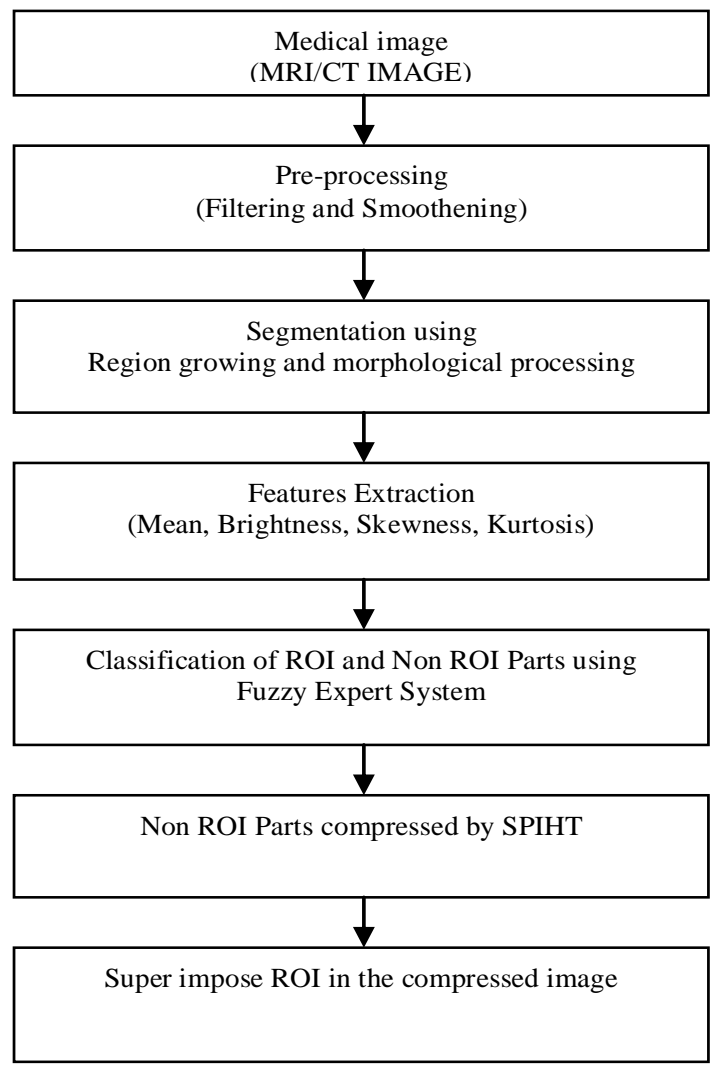

(a)

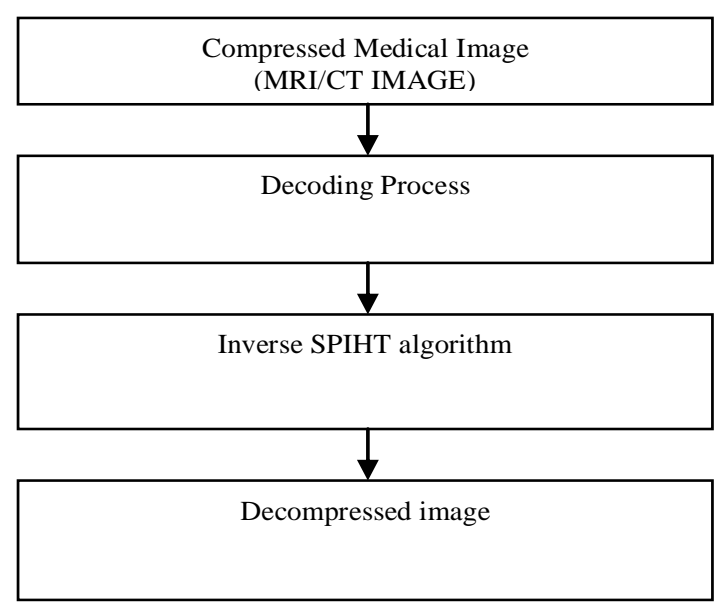

(b)

Figure 1. Block diagram of (a) encoder and (b) decoder.

The degree of compression of background has higher precision than ROI. The several lossless (with exact reconstruction) and lossy (with some information loss) compression techniques have been proposed in different research fields (Wang et al., 2004; Shaou et al., 2007; Kaluanpur et al., 2000). The wavelet compression methods were superior to DCT methods (Shaou et al., 2009). Set Partitioning In Hierarchical Trees (Said et al.,
1996) and JPEG2000 (Taubman et al., 2001; Bartrina et al., 2011; Bradley and Stentiford, 2002) has been proven that algorithms achieved good results for compression of digital images (Arokiapriya et al., 2011). The Maxshift method (Pablo et al., 2008) allowed the ROI compression. Another approach employed low scaling values by modifying the quantization step size of the coefficients at the encoder side (Penedo et al., 2005). Hang et al. (2006) proposed an efficient compression strategy for digital echocardiography using modified SPIHT, which is used to compress tissue data; while a lossless compression strategy based on integer wavelet transform is used to compress doppler data. Somasundaram and Palaniappan (2011) presented adaptive low bit rate facial feature enhanced residual image coding method using SPIHT. Lee (2003) proposed region-based and scalable image compression by wavelet localization. Wang et al. (2004) presented objective methods for assessing perceptual image quality. Dilmaghani et al. (2003) proposed multi rate/resolution control in progressive medical image transmission for the region of interest (ROI). Liu et al. (2002) proposed a lossy-to-lossless region of interest (ROI) compression scheme. Taubman, (2000) presented a new image compression algorithm based on independent embedded block coding with optimized truncation of the embedded bit-streams (EBCOT). Penedo et al. (2003) presented region-based wavelet coding methods for digital mammography. Liu et al. (2002) proposed a new method for chromosome image compression based on an important characteristic of the images. Both Doukas et al. (2005), and Doukas and Maglogiannis (2007) presented medical image compression using wavelet transform on mobile devices with ROI coding support. In the previous work, ROI parts are compressed, this may result in information loss in the medical images. In the proposed method, Fuzzy logic is used to classify ROI and Non ROI. The automatic algorithm is developed to detect the $\mathrm{ROI}$ and communicate it with the human technologist. SPIHT is used for compressing Non ROI parts. The ROI part is superimposed with the compressed Non ROI parts of an image. Finally, PSNR, the compression rate and the compression and decompression time are compared for the proposed and the existing methods in the literature survey. The outcome of this research will throw light on the most effective compression technique for various categories of images.

\section{PROPOSED METHOD}

Pre-processing is done for getting enhanced image using filtering and smoothening processes, the two different clusters (ROI and Non ROI) are obtained by segmentation process. SPIHT is applied for lower energy clusters (Non ROI part of an image) and ROI parts are not compressed by which medical information maintained. In the reconstruction part, higher energy cluster (ROls) is super imposed on compressed lower energy cluster by fusion technique. 


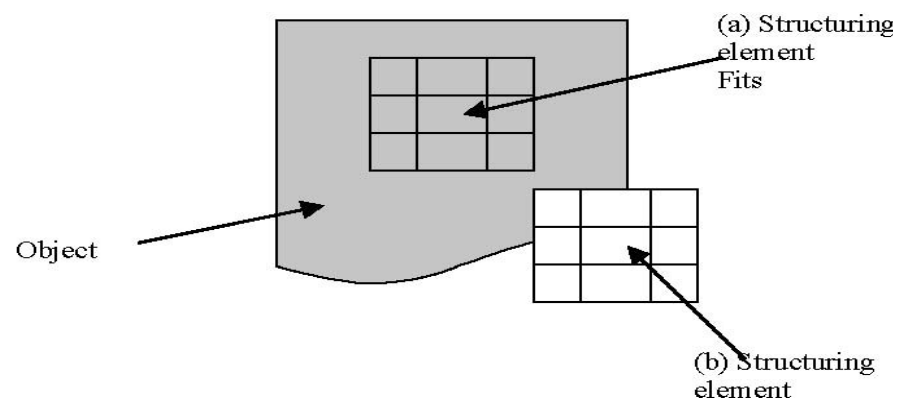

Figure 2. Concept of structuring element fitting and not fitting.

The flow diagram of proposed method is shown in Figure 1a and $1 b$.

\section{Algorithm}

Step 1: Read the medical image.

Step 2: Preprocessing is done for getting enhanced image using filtering and smoothening processes.

Step 3: Segmentation is done using region growing and morphological processing.

Step 4: An important features are extracted from the segmented image.

Step 5: ROI parts is separated using fuzzy logic.

Step 6: SPIHT algorithm is used to compress Non ROI parts of an image.

Step 7: Fusion of both $\mathrm{ROI}$ and Non ROI parts.

Step 8: Decoding process is done.

Step 9: Apply inverse SPIHT algorithm.

Step 10: Evaluate the performance of the proposed compression algorithm.

\section{Pre-processing}

The medical image pre-processing technique is used to improve the image quality and make it ready for further processing by removing noise and unwanted parts in the image. Two stages are involved in the pre-processing. They are: 1) Filtering and 2) Smoothening.

First convert color image into gray scale image. Median filter is used to remove random noise excellently. The smoothing is performed by $5 \times 5$ Gaussian convolution masks. It results in getting a perfect image. The standard deviation of the Gaussian determines degree of smoothing.

\section{Segmentation}

Segmentation (Bai et al., 2007, Anil., 2000) is the process of subdividing an image into its constituent parts. The proposed approach utilizes region growing followed by morphological processing. The gray scale images undergo morphological processing for segmentation of the abnormal regions.

\section{Region growing method}

The process (Kang et al., 2012; Chung et al., 2012) of dividing an image into smaller regions is based upon some predetermined rule that includes how to group the pixels into one location region.
1. The algorithm starts with the selection of the seed pixel and desired predicate $P\left(R_{1}\right)$ to compare with neighbouring pixels.

2. Then, the initial seed is assigned to region $R_{1}$. Then, the eight neighbouring pixels of the seed pixel are assigned to region $R_{1}$,

3 . If these pixels meet the predicate requirement. The process of comparing the eight neighbouring pixels will be continued until no further pixels can be added to the region.

\section{Morphological processing}

The morphological processing (Juan et al., 2005) is used in the smoothening of objects contour and decomposition of an image into its basic geometrical shapes. The implementation of erosion filter followed by a dilation filter is an opening filter and a closing filter is a dilation filter followed by an erosion filter (Tung et al., 2011; Wang et al., 2007). Edge detection (Aminah et al., 2010; Kun et al., 2007) is obtained by the calculating difference between the dilated image and the original image or the original image and the eroded image.

The $A \oplus B$ represent dilation of an image ' $A$ ' by a structuring element ' $B$ '. The following equation is used to compute the dilation, the structuring element ' $\mathrm{B}$ ' is position so that its origin is placed at pixel co-ordinates $(x, y)$.

$$
g(x, y)=\left\{\begin{array}{l}
1 \text { if 'B' Hits 'A' } \\
0, \text { otherwise }
\end{array}\right.
$$

Repeat above process for all pixel co-ordinates. A new image is created by dilation which shows all location of structuring element origin where an input image is hit by structuring element. The $A \Theta B$ represent erosion of an image ' $A$ ' by a structuring element ' $B$ '. The following equation is used to compute the erosion, the structuring element ' $\mathrm{B}$ ' is position so that its origin is placed at pixel coordinates $(x, y)$.

$$
g(x, y)=\left\{\begin{array}{l}
1 \text { if ' } B \text { ' Fits 'A' } \\
0, \text { otherwise }
\end{array}\right.
$$

Repeat above process for all $x$ and $y$ or pixel co-ordinates. A new image is created by dilation which marks all location of structuring element origin where an input image is fitted by structuring element. A morphological processing operator uses a structuring element $B$ to process an image as shown in Figure 2.

There are two possibilities when the structuring element scans over an element in the image, the possibilities are the structuring element completely fits inside the object (Figure 2a) and does not fit inside the object (Figure 2b). Here the morphological processing is used to get the region perfectly.

\section{Features extraction}

Feature extraction (Padma et al ., 2011) is transforming the input data into the set of features and reduces the dimension of an image instead of the full size input. The features are mean, brightness and further characterization of the data includes skewness and kurtosis. The formula for skewness and kurtosis are given below for univariate data $Y_{1}, Y_{2}, \ldots, Y_{N}$,

$$
\text { Skewness }=\frac{\sum_{i=1}^{N}\left(Y_{i}-\bar{Y}\right)^{3}}{(N-1) S^{3}}
$$

Kurtosis $=\frac{\sum_{i=1}^{N}\left(Y_{i}-\bar{Y}\right)^{4}}{(N-1) S^{4}}$ 


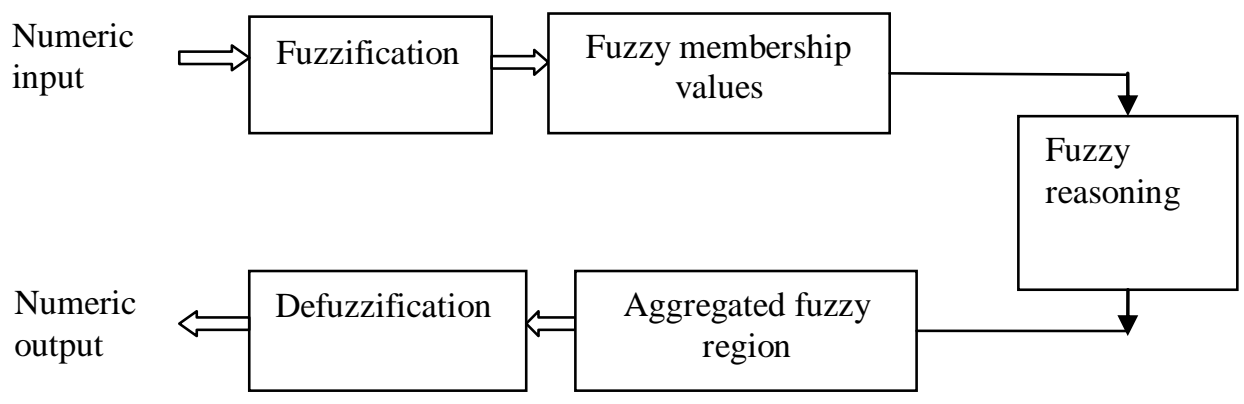

Figure 3. Fuzzy logic theorem in image processing.

Where $\bar{Y}$ is the mean, $S$ is the standard deviation, and $N$ is the number of data points. These features are given as input to fuzzy logic system.

\section{Classification}

Classification is decision-theoretic approaches to the identify cancer and non cancer region of images based on the geometric features sets.

\section{Fuzzy logic}

Fuzzy logic (Renato et al., 2006; Watada et al., 2009) is an extension of Boolean logic that handles the concept of partial truth, which lies between completely true and completely false. The fuzzy logic concept in image processing is shown in Figure 3.

Fuzzy expert systems (Siller et al., 2005) accept inputs numbers and convert them into linguistic values which are then manipulated by if-then rules given by a human expert. After defining the rules and membership functions, the application of this knowledge to the input variables to compute the values of the output variables is studied. The operation of the expert system proceeds as follows.

1. Fuzzification: It is the process of finding the degree of membership of a linguistic variable according to an input value given by the user. The degree of truth for each rule antecedent is calculated by applying the membership functions to the input values.

2. Inference: The if-then rules are implemented and the truth value of each rule is computed using the truth values of the linguistic values. The min and product are commonly used inference rules. The truth value of the implemented rule or the original value of the membership function determines the output membership function in min inferencing. In product inferencing, by the rule premise's truth value scales the output membership function. Min inferencing is used in our method.

3. Composition: For each output linguistic variable, single fuzzy subset is formed by combining all of the fuzzy subsets together. The max and sum methods are commonly used. In max composition, the fuzzy sets for the output variable are ORed with each other so that the maximum value from all the fuzzy subsets is used (fuzzy logic OR). In sum composition, the fuzzy subsets associated with the output value are summed to generate a single fuzzy subset. Max composition is used in or method.

4. Defuzzification: The process in which a scalar value is obtained from the fuzzy output where the set aggregates into a single value.
The centroid and maximum methods are commonly common techniques. In the centroid method, the value of the center of gravity of the membership function for the fuzzy value is found by computing the scalar value of the output variable. The general formula is given below

$$
X(\text { centroid })=\frac{\int_{b}^{a} x \mu(x) d x}{\int_{b}^{a} \mu(x) d x}
$$

where $[a, b]$ is the interval of the aggregated membership function. The RONI membership function is obtained implementing this rule as follows

$$
\mu_{R O N I}\left(p_{i j}\right)=\max \left(\mu_{B}\left(p_{i j}\right) \cdot \mu_{R 1}\left(p_{i j}\right) \cdot \mu_{R 2}\left(p_{i j}\right)\right.
$$

The complement of RONI gives the Region of Interest. For a given image I, after obtaining $\mu_{\mathrm{ROI}}\left(\mathrm{p}_{\mathrm{ij})}\right.$ and $\mu_{\mathrm{C}}\left(\mathrm{p}_{\mathrm{ij})}\right.$ to decide if the image has to be analyzed, and the membership function with which this analysis has to be performed, the fuzzy rule is applied. FCLASS is used to derive fuzzy classification rules from a set of data that can be separated in different crisp classes. The fuzzy rules describing the data are of the form:

If $x_{1}$ is/ 1 and $x_{2}$ is/ 2 and ... and $x_{n}$ is/ n then pattern $\left(x_{n}\right)$ belongs to class $\mathrm{C}$,

where $/ \sim 1, \ldots, / \sim n$ are fuzzy sets. The task of the FCLASS model is to discover these rules, and to learn the shape of the membership functions. The patterns are vectors $x=\left(x_{1} \ldots \ldots x_{n}\right)$ and a class $C$ is a (crisp) subset .The pattern feature values are represented by fuzzy sets and the classification is described by a set of linguistic rules. We allowed the system to create a maximum of six rules using the "best" rule learning algorithm.

if mean is sm and brightness is md and skewness is sm and kurtosis is sm, then ROI

if mean is sm and brightness is sm and skewness is sm and kurtosis is sm, then ROI

if mean is md and brightness is sm and skewness is 19 and kurtosis is 19 , then ROI

if mean is $\mathrm{lg}$ and brightness is sm and skewness is 19 and kurtosis is Ig, then $\mathrm{ROI}$

if mean is md and brightness is sm and skewness is md and kurtosis is Ig, then ROI

if mean is 19 and brightness is md and skewness is 19 and kurtosis is $\lg$, then ROI

$$
\text { *sm- small, md- medium, lg-large }
$$




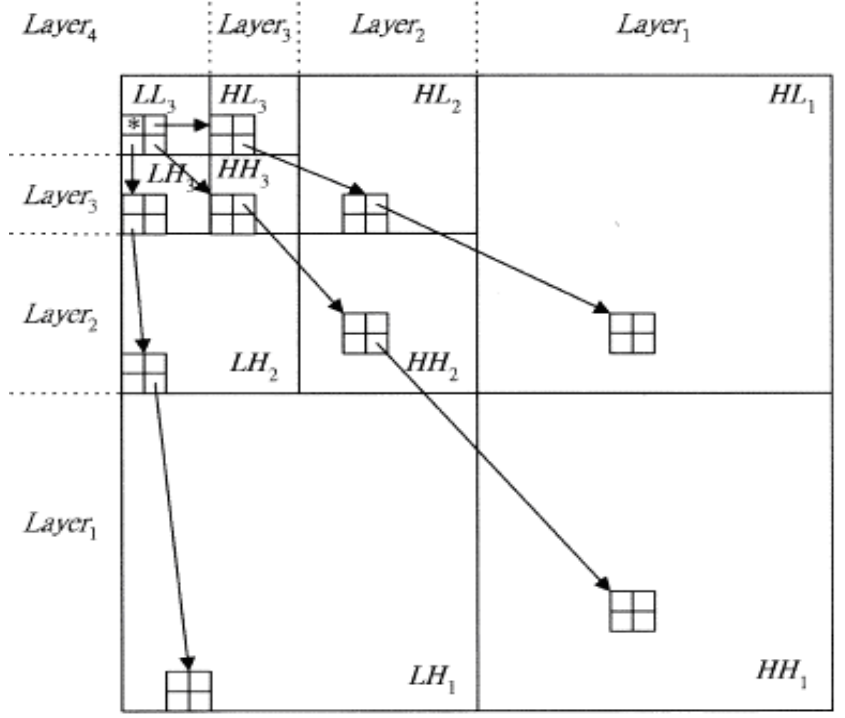

Figure 4. Structure of SPIHT encoding method.

This system reached a classification rate of $99 \%$ on the test set of the data using six rules and four inputs.

ROI: If $\forall\left(p_{i j}\right) \in I, \mu_{R O I}\left(p_{i j}\right)>\mu_{C}\left(p_{i j}\right)$

then I is analyzed, else I is rejected.

\section{Non ROI based compression}

SPIHT is the near lossless compression algorithm which is applied to Non ROI parts of an image.

\section{Set partitioning in hierarchal trees}

Recently, wavelets (Maglogiannis et al., 2009; Grgic et al ., 2001,Talukder and Harada, 2007) have been used frequently in medical image processing. The biorthogonal wavelet transform is taken in this method because it is symmetric, almost orthogonal and gives the best results for medical images. The image is decomposed into different frequency components. 1-D DWT is applied first along the rows of the image and along the columns, thereafter the results are decomposed. This operation results in four decomposed sub band images referred to as low-low (LL), low-high $(\mathrm{LH})$, high-low $(\mathrm{HL})$, and high-high $(\mathrm{HH})$.

SPIHT (Amar et al., 2005; Penedo et al., 2005; Ansari and Anand, 2009) is a medical image coder that exploits the inherent similarities across the sub bands in an integer wavelet decomposition of an image.

Step 1: The original image is decomposed into sixteen sub bands which are shown in Figure 4. By ordering the wavelet coefficients with respect to magnitude. First the most significant bits are transmitted and then the next bit plane follows till the lowest bit plane is reached. According to its significance, the information's are arranged into three types. They are: LIS (list of insignificant sets), LIP (list of insignificant pixels) and LSP (list of significant pixels).

Step 2: The significant coefficients are found by passing the integer wavelet transform coefficients through sorting pass and only the significant coefficient are encoded.
Step 3: The significant coefficients are passed to the refinement pass, the reconstructive value $(R n=R n-1 / 2)$ and threshold $(T n=T n$ $1 / 2$ ) values are decreased in next iteration.

Step 4: Finally, transmit bit stream along with sign bit. The rate can be controlled by using this algorithm. Till the desired distortion value is reached. The same number of lists is replicated by the SPIHT decoder algorithm and also the ordering information is recovered easily.

\section{Quality measures}

The quality of the image (Wang et al., 2004, Schaefer et al., 2005) such as mean square error (MSE) and peak to signal to noise ratio (PSNR), time period and compression ratio are measured at the output of the decoder.

\section{Peak signal noise ratio (PSNR)}

The peak signal-to-noise ratio is defined as the ratio between signal variance and reconstruction error variance. MSE is calculated as:

$\mathrm{MSE}=\sigma_{\mathrm{q}}{ }^{2}=\frac{1}{M * N} \sum_{i=1}^{M} \sum_{j=1}^{N}\left[\mathrm{X}_{i j}-\mathrm{Y}_{i j}\right]^{2}$

where the sum over i,j denotes the sum over all pixels in the image and $M^{*} N$ is the number of pixels in each image. $X_{i j}$-original image, $Y_{\mathrm{ij}}$-Reconstructed image. The PSNR in terms of decibels $(\mathrm{dBs})$ is given by:

$$
\mathrm{PSNR}=10 \log _{10}\left(\frac{255^{2}}{M S E}\right)
$$

Larger PSNR indicate a smaller difference between the original (without noise) and compressed image. This is the most widely used objective image quality/ distortion measure.

\section{Time period}

The time period is defined as the time taken by each method to undergo compression and decompression stages involved in the algorithm.

\section{Compression ratio}

The capability of compression system is characterized by compression ratio which is calculated as

$$
\text { Compression ratio }=\frac{\text { Size of the original image }}{\text { Size of the compressed image }}
$$

\section{RESULTS AND DISCUSSION}

The real images acquired from patients were tested by proposed algorithm. The size of each medical image was $256 \times 256$ pixels. The simulated output of proposed method is discussed below; Figure 5 shows the input images for the compression. Figures 6, 7, 8 and 9 shows the output of pre-processing stages of original images. Figure 10 shows ROI parts of a pre-processed image 


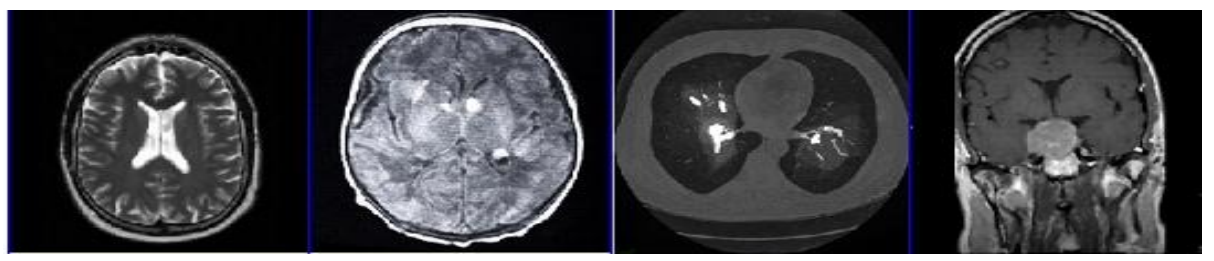

Figure 5. Input medical images.

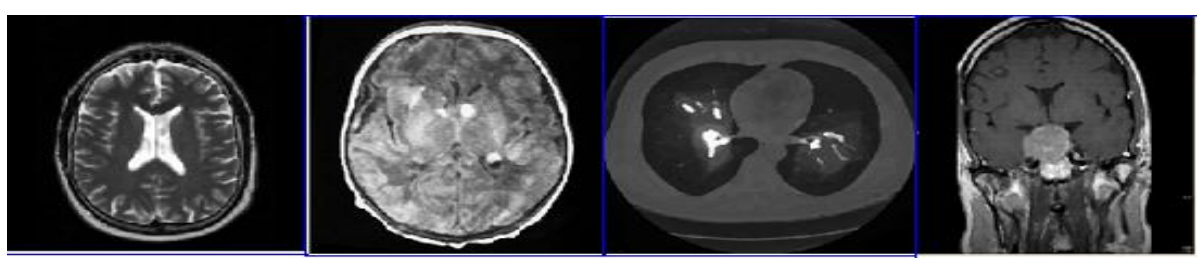

Figure 6. Filter images.

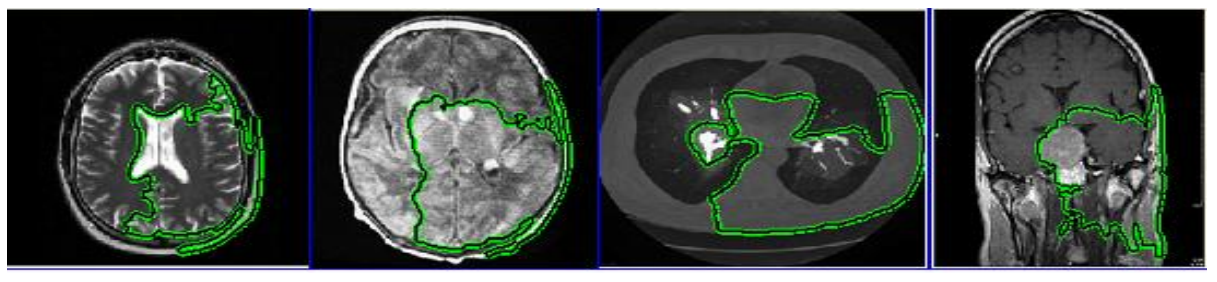

Figure 7. Region growing part of medical images.

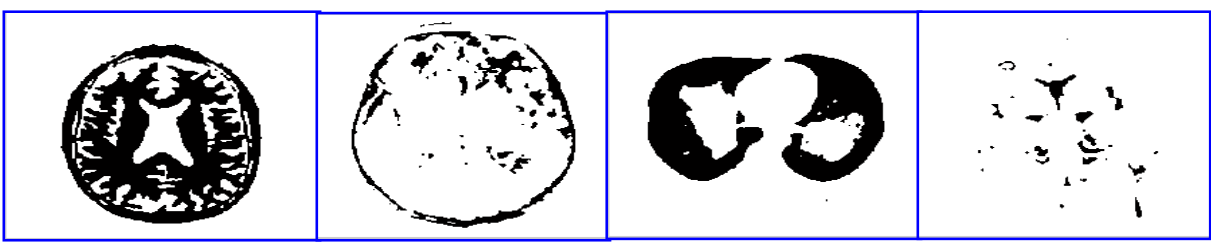

Figure 8. Smoothened medical images.

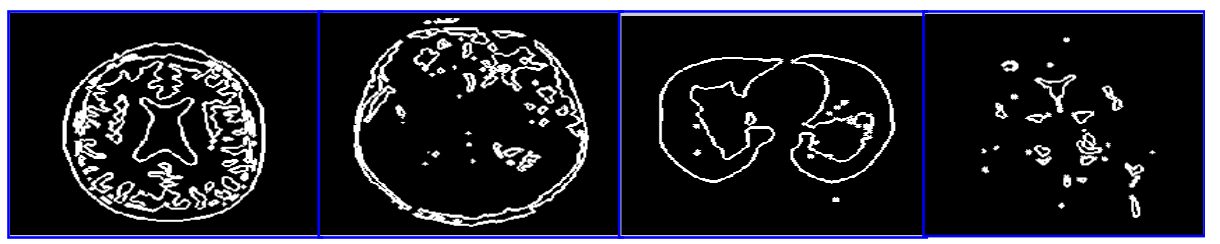

Figure 9. Dilated medical images using morphological processing.

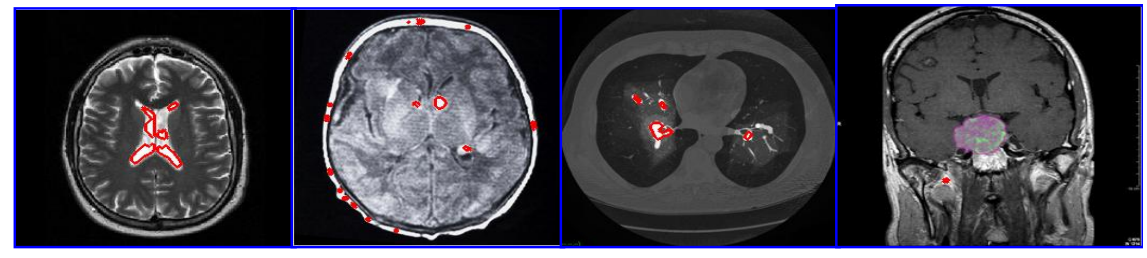

Figure 10. ROI part of medical images using fuzzy logic. 


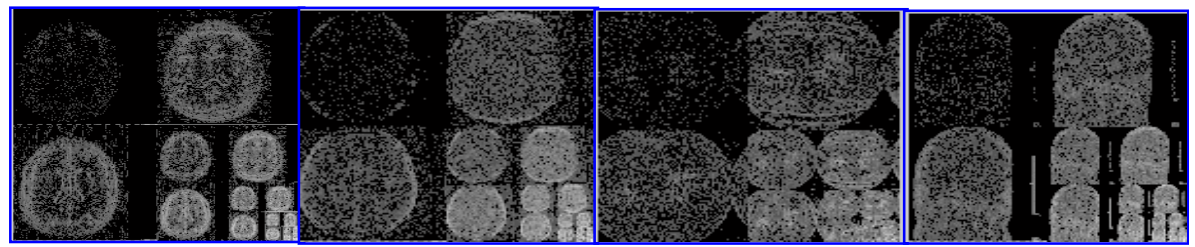

Figure 11. Fourth level decomposition of medical images.

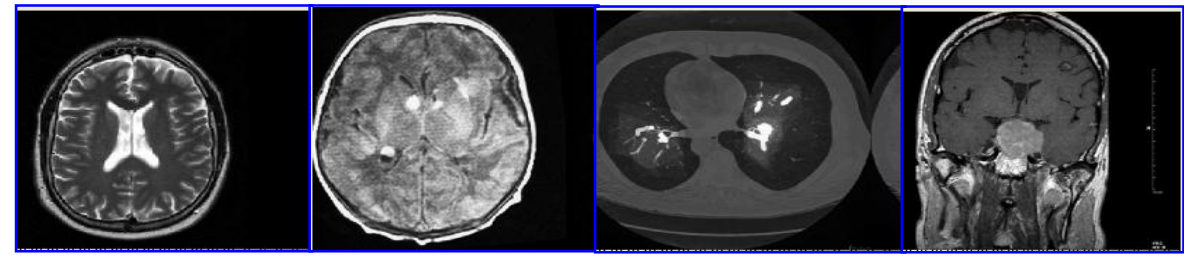

Figure 12. Reconstructed medical images.

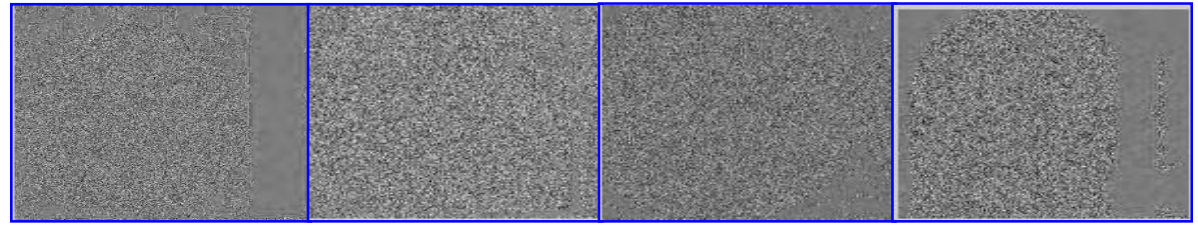

Figure 13. Error images.

using fuzzy logic; Figure 11 shows decomposition of medical images; while Figures 12 and 13 show reconstructed and difference images.

The quality of the medical images is improved by proposed algorithm. The performance of the proposed algorithm are evaluated in terms of quality measures such as MSE, PSNR, CR, Encryption time and Decryption time results for different medical images and is tabulated as follows; The six medical images with different sizes and types are taken for an experiment. The comparison of quality measures of proposed method is shown in Table 1 and Figure 14 compared with other lossless image coders (Chen, 2007). The calculation of PSNR should take both of the preprocessing and compression into account. The PSNR value after preprocessing is $27.1 \mathrm{db}$ and after compression is $60 \mathrm{db}$. Table 2 shows the PSNR value of the medical image after preprocessing and compression process. From Table 1, it is observed that the PSNR values of the proposed compression technique provides significant PSNR value for the standard medical images such as MRI, CT, Ultrasound. When compared with the existing method (Guoli et al., 2005). For instance, when considering the ultrasound liver image with size of $619 \times 482$, the PSNR value and compression rate of the existing compression approach are around $45 \mathrm{db}$ and 2 whereas the PSNR value and compression rate of the proposed method are around $60 \mathrm{db}$ and 4 . Similarly, for other standard images like MRI and CT, the PSNR values and compression rate obtained for the proposed method are observed to be higher than the existing methods compression.

\section{Conclusion}

The compression ratio is increased by the existing compression schemes only by reducing the quality of the medical image. Near lossless compression algorithms were used in this paper. This works well for the images of different sizes. For compression of the segmented non $\mathrm{ROI}$ region lossless compression is used. The advantages of the SPIHT algorithm is that, it does not require codebook design and it is not limited in the size of the blocks used for classification. Moreover for a given bpp, the implicit size of the SPIHT codes extends over the support of the entire image. Finally fusion and decompression are done to the images. Image fusion will join $\mathrm{ROI}$ and Non-ROI parts of an image and hence there is no loss of information. Specifically, for the ultrasound liver image with size of $619 \times 482$, the PSNR is around 60 $\mathrm{dB}$ inferior to that of the SPIHT and the EBCOT algorithms respectively. These, results proved to have better compression performance provided by $\mathrm{ROI}$ based 


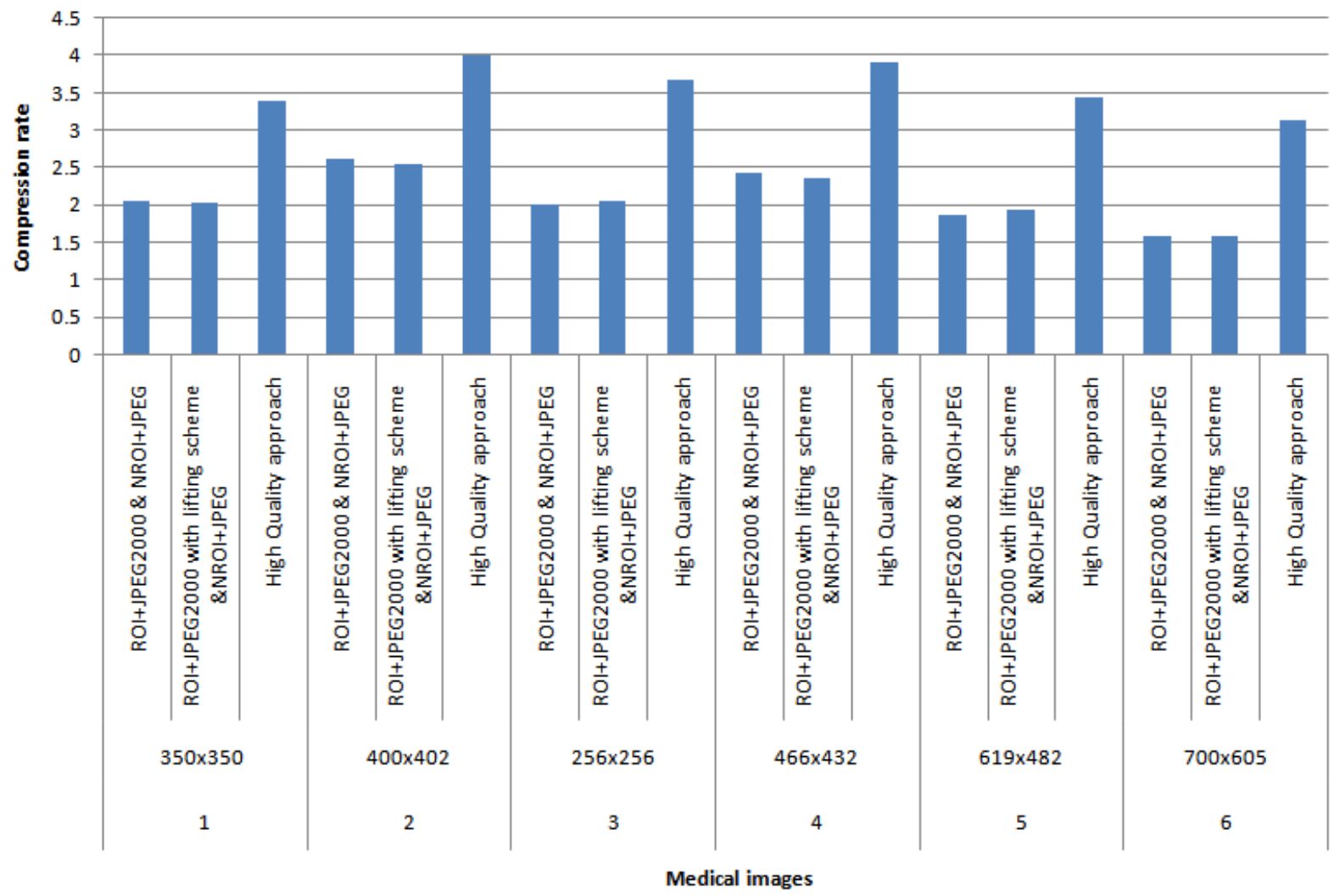

Figure 14. Performance analysis of compression rate for different methods.

Table 1. Performance evaluation in terms of quality measures for different medical images.

\begin{tabular}{|c|c|c|c|c|c|c|}
\hline S.No & $\begin{array}{c}\text { Medical image } \\
\text { size }\end{array}$ & Methodology & CR & PSNR & $\begin{array}{c}\text { Compression } \\
\text { time }\end{array}$ & $\begin{array}{c}\text { Decompression } \\
\text { time }\end{array}$ \\
\hline \multirow{3}{*}{1} & \multirow{3}{*}{$350 \times 350$} & ROI+JPEG2000 \& NROI+JPEG & 2.06 & 36.57 & 0.52 & 0.89 \\
\hline & & ROI+JPEG2000 with lifting scheme \&NROI+JPEG & 2.03 & 25.09 & 0.13 & 0.55 \\
\hline & & High quality approach & 3.4 & 60.02 & 2.14 & 1.34 \\
\hline \multirow{3}{*}{2} & \multirow{3}{*}{$400 \times 402$} & ROI+JPEG2000 \& NROI+JPEG & 2.61 & 33.67 & 0.24 & 1.63 \\
\hline & & ROI+JPEG2000 with lifting scheme \&NROI+JPEG & 2.54 & 26.68 & 0.18 & 0.99 \\
\hline & & High quality approach & 4 & 51.73 & 2.48 & 1.64 \\
\hline \multirow{3}{*}{3} & \multirow{3}{*}{$256 \times 256$} & ROI+JPEG2000 \& NROI+JPEG & 2.01 & 34.84 & 0.31 & 1.6 \\
\hline & & ROI+JPEG2000 with lifting scheme \&NROI+JPEG & 2.04 & 25.7 & 0.32 & 1.39 \\
\hline & & High quality approach & 3.68 & 59.09 & 2.41 & 1.56 \\
\hline \multirow{3}{*}{4} & \multirow{3}{*}{$466 \times 432$} & ROI+JPEG2000 \& NROI+JPEG & 2.43 & 35.73 & 0.19 & 1.01 \\
\hline & & ROI+JPEG2000 with lifting scheme \&NROI+JPEG & 2.35 & 13.85 & 0.1 & 1.39 \\
\hline & & High quality approach & 3.9 & 54.72 & 2.07 & 1.37 \\
\hline \multirow{3}{*}{5} & \multirow{3}{*}{$619 \times 482$} & ROI+JPEG2000 \& NROI+JPEG & 1.87 & 42.14 & 0.02 & 0.09 \\
\hline & & ROI+JPEG2000 with lifting scheme \&NROI+JPEG & 1.94 & 36.49 & 0.06 & 0.28 \\
\hline & & High quality approach & 3.45 & 60.59 & 2.03 & 1.41 \\
\hline \multirow{3}{*}{6} & \multirow{3}{*}{$700 \times 605$} & ROI+JPEG2000 \& NROI+JPEG & 1.57 & 42.96 & 0.08 & 0.27 \\
\hline & & ROI+JPEG2000 with lifting scheme \&NROI+JPEG & 1.57 & 15.21 & 0.08 & 1.39 \\
\hline & & High quality approach & 3.14 & 59.14 & 2.2 & 1.4 \\
\hline
\end{tabular}


algorithms, in terms of higher PSNR and visual quality. Due to the increase in use of digital techniques in Telemetry, the amount of digital imaging data for storage or transmission grows rapidly. Efficient medical image compression methods help to alleviate this problem by increasing the compression ratio without degrading its original quality.

\section{REFERENCES}

Amar CB, Zaied M, Alimi A (2005). Beta wavelets Synthesis and application to lossy image compression. Adv. Eng. Softw. 36:459474.

Aminah AM, Wan Eny Z, Wan Abdul R, Arsmah I, Rozi M, Siti Salmah Y, Abdul KJ (2010). Region and Boundary Segmentation of Microcalcifications using Seed-Based Region Growing and Mathematical Morphology. Proced. Soc. Behav. Sci. 8:634-639.

Anil KJ (2000). Fundamentals of Digital Image Processing. Prentice Hall.

Ansari MA, Anand RS (2009). Context based medical image compression for ultrasound images with contextual set partitioning in hierarchical trees algorithm. Adv. Eng. Soft. 40(7):487-496.

Arokia Priya R, Narote SP, Patil AV (2011). Dual Tree Wavelet Transforms in Image Compression. Int. J. Eng. Sci. Res (IJESR). 1(1):223-234.

Bai X, Jin JS, Feng D (2007). Segmentation based multilayer diagnosis lossless medical image Compression. Proc. ACM Int. Conf. 100:9-14.

Bradley AP, Stentiford FWM (2002). JPEG 2000 and Region of Interest Coding in Digital Image Computing. Techniques and Applications (DICTA002), Melbourne, Australia. pp. 303-308.

Chung-Chia K, Wen-June W, Chung-Hao K (2012). Image segmentation with complicated background by using seeded region growing. AEU - Int. J. Elec. Comm. 66(9):767-771.

Dilmaghani R, Ahmadian A, Ghavami M, Oghabian M, Aghvami $H$ (2003). Multi rate/resolution control in progressive medical image transmission for the region of interest (ROI) using EZW. Proc. IEEE $25^{\text {th }}$ Annu. Int. Conf. Eng. Med. Biol. Soc. pp. 818-820.

Doukas C, Maglogiannis I (2007). Region of Interest Coding Techniques for Medical Image Compression. IEEE Trans. Eng. Med. Biol. Mag. 26(5):29-35.

Doukas C, Maglogiannis I, Kormentzas G (2005). Medical Image Compression using Wavelet Transform on Mobile Devices with ROI coding support. Conf Proc. IEEE Eng .Med. Biol. Soc. 4:3779-3784.

Grgic S, Grgic M, Zovko-cihlar B (2001). Performance analysis of image compression using wavelets. IEEE Trans. Ind. Electr. 48:682695.

Guoli L, ZHANG J, WANG Q, Cungang H, DENG N, Jianping L (2005). Application of Region Selective Embedded Zero tree Wavelet Coder in CT Image Compression. IEEE Eng. Med. Biol. 27 ${ }^{\text {th }}$ Annual Conference, Shanghai, China pp. 6591-6594.

Hanga X, Neil LG, James DT (2006). Compression of pre-scan converted echocardiographic video using wavele packet and integer wavelet transforms. Ima. Vis. Comp. 24(9):915-925.

Joan B-R, Joan S-S, Francesc A-L (2011). JPEG 2000 ROI coding through component priority for digital mammography. Com.Vis. Image Understanding 115(1):59-68.

Juan I, Pastore, Emilce, Moler G, Virginia, Ballarin L (2005). Segmentation of brain magnetic resonance images through morphological operators and geodesic distance. Dig. Sig. Proc. 15(2):153-160.

Kaluanpur A, Neklesa VP, Taylor CR, Daftary AR, Brink JA (2000). Evaluation of JPEG and wavelet compression of body CT images for direct digital teleradiologic transmission. Radiology 217:772-779.

Kun W, Jianhua W, Zhaoyu P, Li G, Liqun GAO (2007). Edge Detection Algorithm for Magnetic Resonance Images Based on Multiscale Morphology. IEEE International Conference Control and Auto. Guangzhou, China pp. 2437-2440.

Lee MS (2003). Region-based and scalable image compression by wavelet localization. Proc. 7th Int. Symp. Signal Process. Appl. 2:451-454.
Liu L , Ha J, Xiong Z , Wu Q, Castleman K (2002). Lossy-to-lossless ROI coding of chromosome images using modified SPIHT and EBCOT. Proc. IEEE Int. Symp. Biomed. Imag. pp. 317-320.

Liu L, Fan G (2003). A new JPEG2000 region-of-interest image coding method: Partial significant bitplanes shift. IEEE Sig. Process. Lett. 10(2):35-39.

Liu Z, Ha J, Xiong Z, Wu Q, Castleman K (2002). Cascaded differential and wavelet compression of chromosome images. IEEE Trans. Biomed. Eng. 49(4):372-383.

Maglogiannis I, Doukas C, Kormentzas G, Pliakas T (2009). WaveletBased Compression With ROI Coding Support for Mobile Access to DICOM Images Over Heterogeneous Radio Networks. IEEE Trans. Inf. Tech. Biomed. 13(4):458-466.

Pablo G, Tahoces A, Ramon Varela A, Marıa J, Lado B (2008). Image compression: Maxshift ROI encoding options in JPEG2000. Comp. Vis. Image Understanding. 109:139-145.

Padma A, Sukanesh R (2011). Texture feature based analysis of segmenting soft tissues from brain CT images using BAM-type artificial neural network. J. Inf. Eng. Appl. 1(4):34-43.

Penedo M, Pearlman WA, Tahoces PG, Souto M, Vidal JJ (2003). Region-based wavelet coding methods for digital mammography. IEEE Trans. Med. Imag. 22(10):1288-1296.

Penedo M, Souto M, Tahoces PG, Carreira JM, Villalon J, Porto G (2005). Evaluation of JPEG2000 and object-based SPIHT lossy compression on digitized mammograms. Radiology 237:450-457.

Renato C, Maria A, Gil, Henk A, Kiers L (2006). The Fuzzy Approach to Statistical Analysis. Comp. Stat. Data. Anal. 51(1):1-14.

Said A, Pearlman WA (1996). A new fast and efficient image codec based on Set Partitioning in Hierarchical Trees. IEEE Trans. Circ. Syst. Video Technol. 6:243-250.

Schaefer G, Starosolski R, Zhu S Y (2005). An evaluation of Lossless compression algorithms for medical infrared images. Proc. IEEE Eng. Med. Biol. Conf. pp. 1673-1676.

Shaou-Gang M, Fu-Sheng K, Shu-Ching C (2009). A Lossless Compression Method for Medical Image Sequences Using JPEG-LS and Interframe Coding. IEEE Trans.Inf. Tech. Biomed. 13(5).

Shaou-Gang M, Shih TC (2007). Automatic quality control for waveletbased compression of volumetric medical images using distortion constrained adaptive vector quantization. IEEE Trans. Med. Imag. 23(11):1417-1429.

Somasundaram K, Palaniappan N (2011). Adaptive low bit rate facial feature enhanced residual image coding method using SPIHT for compressing personal ID images. AEU - Int. J. Elec. Comm. 65(6):589-594

Talukder KH, Harada K (2007). Haar Wavelet Based Approach for Image Compression and Quality Assessment of Compressed Image. IAENG Int. J. Appl. Math. 36(1):6-14.

Taubman D (2000). High performance scalable image compression with EBCOT. IEEE Trans. Image Process. 9(7):1158-1170.

Taubman DS, Marcellin MW (2001). JPEG 2000 Image Compression Fundamentals, Standards and Practice, Kluwer Academic Publishers, Boston 1:34-42.

Tung-Ching S, Ming-Der Y, Tsung-Chiang W, Ji-Yuan L (2011). Morphological segmentation Based on edge detection for sewer pipe defects on CCTV images. Exp. Sys. Appl. 38(10):13094-13114.

Wang A, Bovik A. C, Sheikh H. R, Simoncelli E. P(2004). Image quality assessment: From error visibility to structural similarity. IEEE Trans. Image Process. 13(4):600-612.

Wang Z, Leung CS, Zhu YS, Wong TT (2004). Data compression with spherical wavelets and wavelets for the image-based relighting. Comput. Vis. Image Und. 96:327-344.

Watada J, Shuming Wang, Pedrycz W (2009). Building ConfidenceInterval-Based Fuzzy Random Regression Models. IEEE Trans. Fuzzy Syst. 17(6):1273-1283.

Yen-Yu Chen (2007). Medical image compression using DCT-based subband decomposition and modified SPIHT data organization. Int. J. Med. Inf. 76(10):717-725. 